\title{
Discovery of New Filicinic Acid-based Meroterpenoids from Hypericum japonicum
}

\section{Linzhen $\mathrm{Hu}^{1}$, Yonghui Zhang ${ }^{2}$, Yong Chen ${ }^{1}$, Zhenzhen Wang ${ }^{2}$, Rongrong Wu${ }^{1}$, Shuying Tian ${ }^{1}$, Yongbo Xue ${ }^{2}$}

${ }^{I}$ School of Life Science, Hubei University, China, ${ }^{2}$ School of Pharmacy, Tongji Medical College, Huazhong University of Science and Technology, China

Natural products have been utilized sophisticatedly as traditional medicines for millennia ${ }^{1}$. In recent decades, an increasing discovery of natural products provided prolific sources of new chemical entities served as therapeutic drugs or lead compounds ${ }^{2}$. The genus Hypericum (Guttiferae) has triggered wide investigations of the scientific community ${ }^{3}$, owing to phloroglucinol derivatives possessing diversiform structures, and appealing bioactivities such as antidepressant ${ }^{4}$, antibacterial $^{5}$, anticancer ${ }^{6}$, and antiviral ${ }^{7}$ properties. In our continuous course of systematic study on the genus Hypericum $^{8,9}$, one pair of enantiomers $( \pm)$-hyperjaponols $\mathrm{H}(\mathbf{1} \mathbf{a} / \mathbf{1} \mathbf{b})$, two pairs of epimers hyperjaponols J-M (3-6), as well as hyperjaponol I (2), six new hetero-Diels-Alder adducts of polymethylated phloroglucinol and sesquiterpene motifs giving shape to filicinic acid ${ }^{-}$based meroterpenoids, along with 6/6/10 ring skeletons (Figure 1), were isolated from the entire herbs of Hypericum japonicum. Their structures with absolute configurations were characterized on the extensive analyses of NMR spectra, ECD calculations, and the experimental ECD data. The aforementioned metabolites exhibited moderate inhibitory effects on lytic replication of Epstein-Barr virus (EBV).

\section{References}

1. Clardy, J.; Walsh, C., Nature 2004, 432, 829-837.

2. Li, J. W. H.; Vederas, J. C., Science 2009, 325, 161-166.

3. Ciochina, R.; Grossman, R. B., Chem. Rev. 2006, 106, 3963-3986.

4. Richard, J. A. Eur. J. Org. Chem. 2014, 2014, 273-299.

5. Oya, A.; Tanaka, N.; Kusama, T.; Kim, S. Y.; Hayashi, S.; Kojoma, M.; Hishida, A.; Kawahara, N.; Sakai, K.; Gonoi, T.; Kobayashi, J., J. Nat. Prod. 2015, 78, 258-264.

6. Zhang,S.; Yin, J.; Li, X.; Zhang, J.; Yue, R.; Diao, Y.; Li, H.; Wang, H.; Shan, L.; Zhang, W., BMC Cancer 2014, 14, 689 $-699$.

7. Singh, I. P.; Bharate, S. B., Nat. Prod. Rep. 2006, 23, 558-591.

8. Hu, L.; Zhang, Y.; Zhu, H.; Liu, J.; Li, H.; Li, X. N.; Sun, W.; Zeng, J.; Xue, Y.; Zhang, Y., Org. Lett. 2016, 18, 2272 -2275 .

9. Zhu, H.; Chen, C.; Yang, J.; Li, X. N.; Liu, J.; Sun, B.; Huang, S. X.; Li, D.; Yao, G.; Luo, Z.; Li, Y.; Zhang, J. ; Xue, Y.; Zhang, Y., Org. Lett. 2014, 16, 6322-6325. 